\title{
CARACTERIZACIÓN FÍSICA DE LOS RESIDUOS SÓLIDOS DE LA CIUDAD DE TEPIC Y SU POTENCIAL ENERGÉTICO
}

\author{
C.E. SALDAÑA, I. P. HERNÁNDEZ, S. MESSINA
}

${ }^{1}$ Universidad Autónoma de Nayarit, Ciudad de la Cultura Amado Nervo, Tepic, Nayarit, C.P. 63190.

${ }^{2}$ Centro de Investigación en Energía UNAM, Privada Xochicalco S/N, Temixco, Morelos, C.P. 62580.

E-mail: paz.rosales@gmail.com

\begin{abstract}
Resumen
Se presenta la caracterización física de los residuos sólidos urbanos (RSU) y la estimación de su potencial energético teórico para su utilización como combustible en un incinerador. La situación en la ciudad de Tepic, en el contexto del manejo y disposición final de residuos, se caracteriza por no tener una cultura de separación y reciclaje además de una recolección ineficiente que provoca que los RSU permanezcan en los domicilios por más de tres días. De las más de 600 toneladas generadas en un día, el material más importante de los RSU son los residuos orgánicos que contribuyen con el 53.77\%, lo cual se transforma en un grave problema ambiental y de salud pública. En este trabajo se plantea aprovechar el valor calorífico de los RSU para su utilización como combustible para generar energía eléctrica. Los resultados indican que a partir de estos desechos se pueden generar más de $600 \mathrm{KWh}$ /día lo que equivale a desplazar más de 1.6 Tm de petróleo o $1920 \mathrm{~m} 3$ de gas natural al mes.
\end{abstract}

\section{Introducción}

La producción de RSU ha aumentado en todo el mundo como consecuencia del incremento de la población, las actividades humanas y el desarrollo de la tecnología. Adicionalmente, la gestión en la disposición y control de los RSU es compleja debido a la variedad y cantidad de desechos, a un sistema de recolección ineficiente, a la inadecuada disposición final, a bajos presupuestos asignados al manejo de los residuos, impactos al ambiente, falta de participación ciudadana, y a los patrones de consumo de la sociedad.

El procesamiento térmico de los RSU utilizado para la reducción en volumen y la recuperación de energía, es un elemento importante en muchos sistemas de gestión integral de residuos sólidos; se puede definir como la conversión de los RSU en productos de conversión gaseosos, líquidos y sólidos, con la subsiguiente emisión de energía en forma de calor. Estudios realizados por Tchobanoglous indican que el contenido energético de los RSU es de $2,816 \mathrm{kcal} / \mathrm{kg}[1]$.

La recolección de los RSU de la ciudad de Tepic se realiza a: 47 rutas diarias en 24 días trabajando en turno matutino, vespertino y nocturno, mismas que se encuentran distribuidas en 239 colonias populares; servicio especial a: 44 poblados, 31 edificios públicos, 5 mercados municipales, 160 escuelas públicas y privadas; también existe un servicio especial a 147 convenios de negocios mediante contrato con la Tesorería. Así, la cantidad de RSU recolectada asciende a más de 600 toneladas diarias que sólo se disponen en el tiradero municipal "el Ixtete" las cuales no reciben ningún tratamiento para su aprovechamiento, como podría ser el reciclaje, el compostaje de la materia orgánica y producción energética utilizando gasificación, pirolisis e incineración.

En este trabajo se presenta la caracterización física de los RSU y la cuantificación del potencial energético (teórico) para su utilización como combustible en procesos de incineración bruta.

\section{Metodología}

Se realizó la caracterización física de los RSU del tiradero el Ixtete de la ciudad de Tepic. Para la composición porcentual de los RSU se empleó el método del cuarteo bajo la norma NMX-AA-015-1985 y NMX-AA-022-1985 $[2,3]$. El procedimiento consistió en tomar una muestra de forma aleatoria de basura y colocarla en una superficie limpia, que tenga un espacio de ancho y largo de cuatro metros. La basura se colocó en el centro dándole movimiento constante por medio de una pala hasta lograr una mezcla homogénea. Después, se retiró cuatro partes opuestas del montón, dejando en el centro por lo menos $50 \mathrm{~kg}$ de basura, la cual se agrupó de acuerdo a la composición de materiales más comunes: materia orgánica, plástico, papel, cartón y otros. En seguida se pesaron los materiales obteniendo una relación de porcentajes de la composición de cada uno de ellos. Los porcentajes de cada material clasificado según datos proporcionados por el Programa Municipal para la Prevención y Gestión Integral de Residuos Sólidos de Tepic para el año 2009 [4] se muestran en la tabla 1. 
En la tabla 2 se muestran las toneladas de RSU recolectadas en el periodo Enero a Junio de 2010.

Tabla 1. Composición de los RSU de la ciudad de Tepic, Nayarit [4]

\begin{tabular}{|c|c|}
\hline Material & Porcentajes \\
\hline Orgánico & 53.77 \\
\hline Plástico & 13.64 \\
\hline Papel & 9.82 \\
\hline Residuos de Jardín & 3.29 \\
\hline Pañal & 6.04 \\
\hline Cartón & 3.9 \\
\hline Vidrio & 2.43 \\
\hline Residuos finos & 1.63 \\
\hline *Otros & 3.08 \\
\hline
\end{tabular}

*Material de construcción, Metal, Aluminio, Unicel, Madera

Tabla 2. Toneladas métricas de RSU recolectadas en el relleno sanitario el Ixtete durante el periodo Ene-Jun de 2010.

\begin{tabular}{|c|c|c|c|c|c|c|}
\hline & ENE & FEB & MAR & ABR & MAY & JUN \\
\hline Toneladas recolectadas & 20,844 & 18,814 & 21,246 & 19,282 & 19,384 & 19,582 \\
\hline
\end{tabular}

Se determinó el valor calorífico, CV (por sus siglas en inglés) de la muestra que contiene residuos de comida, papel, cartón y plásticos a la que se denominará "OPCP" con el método genérico utilizando la sumatoria del producto de los valores genéricos del contenido energético de cada componente multiplicado por su fracción de la muestra de RSU, basándose en los datos de Tchobanoglous [1]. Adicionalmente, se obtuvo el CV de la muestra OPCP con los modelos empíricos de: Dulong, Steuer y Scheurer-Kestner [5].

Tabla 3. Valores genéricos del contenido energético de los RSU.

\begin{tabular}{|c|c|c|c|}
\hline Componente & Intervalo de energía (kcal/kg) & Valor típico de energía (kcal/kg) & Porcentaje \\
\hline Orgánicos & $833-1667$ & 1111 & 53.77 \\
\hline Papel & $2778-4444$ & 4000 & 9.82 \\
\hline Cartón & $3333-4167$ & 3889 & 3.9 \\
\hline Plásticos & $6667-8889$ & 7778 & 13.64 \\
\hline Muestra & $\mathbf{1 7 6 0 . 0 7 - 2 7 0 7 . 7 2}$ & $\mathbf{2 2 0 2 . 7 7}$ & \\
\hline
\end{tabular}

Tabla 4. Datos típicos del contenido de humedad de los RSU.

\begin{tabular}{|c|c|c|c|}
\hline Tipo de Residuo & $\begin{array}{c}\text { Contenido humedad (\%W) } \\
\text { Valor típico }\end{array}$ & Muestra con agua (kg) & Muestra sin agua (kg) \\
\hline Orgánico & 70 & 53.77 & 16.13 \\
\hline Papel & 6 & 9.82 & 9.23 \\
\hline Cartón & 5 & 3.9 & 3.71 \\
\hline Plásticos & 2 & 13.64 & 13.37 \\
\hline \multicolumn{2}{|c|}{ Peso total de la muestra (OPCP) } & 81.13 & 42.44 \\
\hline
\end{tabular}

*Porcentaje en peso de agua de la muestra $\mathrm{OPCP}=47.69 \%$, que equivalen a $38.69 \mathrm{~kg}$ de agua en $\mathrm{OPCP} / 100 \mathrm{~kg}$ de muestra de los RSU.

Con base en los datos generados en las tablas 3 y 4, así como los datos genéricos de la referencia [1] se estimó la composición química de la muestra OPCP de los RSU, con y sin agua, como se presenta en la tabla 5. 
Tabla 5. Estimación de la composición química de la muestra OPCP de los RSU.

\begin{tabular}{|c|c|c|c|c|c|c|c|}
\hline & & \multicolumn{6}{|c|}{ Masa de componente en kg/100kg de muestra total de RSU } \\
\hline Tipo de residuo & Peso seco kg & $\mathbf{C}$ & $\mathbf{H}$ & $\mathbf{O}$ & $\mathbf{N}$ & $\mathbf{S}$ & Cenizas \\
\hline Orgánico & 16.13 & 7.74 & 1.03 & 6.06 & 0.42 & 0.07 & 0.81 \\
\hline Papel & 9.23 & 4.02 & 0.55 & 4.06 & 0.03 & 0.02 & 0.55 \\
\hline Cartón & 3.71 & 1.63 & 0.22 & 1.65 & 0.02 & 0.01 & 0.19 \\
\hline Plásticos & 13.37 & 8.02 & 0.96 & 3.05 & - & - & 1.34 \\
\hline Total (kg) & $\mathbf{4 2 . 4 4}$ & $\mathbf{2 1 . 4 1}$ & $\mathbf{2 . 7 6}$ & $\mathbf{1 4 . 8 2}$ & $\mathbf{0 . 4 6}$ & $\mathbf{0 . 1 0}$ & $\mathbf{2 . 8 9}$ \\
\hline $\begin{array}{c}\text { Porcentaje en peso de la } \\
\text { muestra OPCP con agua. }\end{array}$ & $\mathbf{2 6 . 3 9 \%}$ & $\mathbf{3 . 4 0} \%$ & $\mathbf{1 8 . 2 7 \%}$ & $\mathbf{0 . 5 7 \%}$ & $\mathbf{0 . 1 2} \%$ & $\mathbf{3 . 5 6 \%}$ \\
\hline
\end{tabular}

Se procedió a estimar el contenido energético de los RSU típicos basándose en su composición química y en los modelos empíricos, utilizando las ecuaciones (1), (2) y (3) [5]. Los resultados son mostrados en la tabla 6.

1. Modelo Dulong :

$$
C V=81 C+342.5(H-O / 8)+22.5 S-6(9 H+W)
$$

2. Modelo Steuer:

$$
C V=81(C-3 O / 8)+57(3 \mathrm{O} / 8)+345(H-O / 16)+25 S-6(9 H+W)
$$

3. Modelo Scheurer-kestner:

$$
C V=81(C-3 O / 4)+342.5 H+22.5 S+57(3 O / 4)-6(9 H+w)
$$

donde $\mathrm{CV}=$ valor calorífico $\mathrm{kcal} / \mathrm{kg}, \mathrm{C}=\%$ peso carbono, $\mathrm{H}=\%$ peso hidrógeno, $\mathrm{O}=\%$ peso de oxigeno, $\mathrm{S}=\%$ peso de azufre

Tabla 6. Estimación del valor calorífico de la muestra OPCP de los RSU.

\begin{tabular}{|c|c|}
\hline Modelo & Valor calorífico (kcal/kg) \\
\hline Modelo Dulong & 2052.86 \\
\hline Modelo Steuer & 2285.47 \\
\hline Modelo Scheurer-kestner & 2506.19 \\
\hline
\end{tabular}

\section{Resultados}

Con base en los datos de recolección de RSU de la Tabla 2, se obtiene el valor promedio mensual de 19,858.66 Tm, de los cuales sólo se incineraran el 81.13\% (16,111.334 Tm de OPCP/mes). La incineración de la muestra OPCP nos proporciona $33074.32 \mathrm{Mcal} / \mathrm{mes}$ lo que equivale a $38.44 \mathrm{MWh} / \mathrm{mes}$ (con base al CV del modelo de Dulong). Considerando el factor de conversión de rendimiento eléctrico equivalente de una planta incineradora de España de $50.5 \%$ se tiene una producción de $19.41 \mathrm{MWh} / \mathrm{mes}$ (0.64MWh/día) [6]. Adicionalmente se utilizaron las equivalencias de energía producida en relación al combustible usado [7]. Con base en éstos se obtuvo el desplazamiento de $55 \mathrm{Kg}$ de petróleo o $64 \mathrm{~m}^{3}$ de gas natural al día.

\section{Conclusiones}

Debido a que el CV obtenido es mayor a $2000 \mathrm{kcal} / \mathrm{kg}$ la incineración de los RSU es una alternativa viable para resolver en el corto plazo el grave problema de los residuos en la ciudad de Tepic. Con ésta se elimina la existencia de plagas, malos olores, escurrimientos de lixiviados a mantos acuíferos, se reduce el peso y el volumen de los residuos en los vertederos, se reduce la formación de metano y se elimina el riesgo de combustión espontánea en los vertederos. Adicionalmente, a la producción de energía obtenida por la incineración de RSU, las escorias procedentes de la combustión pueden ser usadas como sub-base de carreteras. Sin embargo, la incineración no resuelve el problema de los residuos y entorpece los programas de minimización, reciclaje y tecnologías limpias. 


\section{Referencias}

[1] TCHOBAnOGLOUS, G., Theisen, H. Y Vigil, S. Gestión Integral de Residuos Sólidos. Traducido de la 1º. Ed. México: McGraw-Hill, Vol. 1, 1997.

[2] NMX-AA-015-1985, Protección al ambiente-contaminación del suelo-residuos sólidos municipales-muestreo-método del cuarteo. Secretaria de Comercio y Fomento Industrial, Dirección General de Normas.

[3] NMX-AA-022-1985, Protección al ambiente-contaminación del suelo-residuos sólidos municipales- selección y cuantificación de subproductos. Secretaria de Comercio y Fomento Industrial, Dirección General de Normas.

[4] Programa Municipal para la Prevención y Gestión Integral de Residuos Sólidos de Tepic, 2009.

[5] Moh'd Abu-Qudais, Hani A. Abu-Qdais. Energy content of municipal solid waste in Jordan and its potential utilization. Energy Conversion and Management, vol.41, pp 983-991, 2000.

[6] Rubio Martín A.,ETSE-electricidad, Estudio técnico de una central eléctrica de incineración de RSU, España, noviembre 2003.

[7] Asociación para el estudio de los recursos energéticos de España (aeren-ASPO-SPAIN) página web (http://www.crisisenergetica.org/staticpages/index.php?page=20040125190131832) (agosto de 2010). 